\title{
A comparison of turbulent pipe, channel and boundary layer flows
}

\author{
J. P. MONTY†, N. HUTCHINS, H. C. H. NG, I. MARUSIC \\ AND M. S. CHONG \\ Department of Mechanical Engineering, University of Melbourne, VIC 3010, Australia
}

(Received 18 November 2008 and in revised form 20 March 2009)

The extent or existence of similarities between fully developed turbulent pipes and channels, and in zero-pressure-gradient turbulent boundary layers has come into question in recent years. This is in contrast to the traditionally accepted view that, upon appropriate normalization, all three flows can be regarded as the same in the near-wall region. In this paper, the authors aim to provide clarification of this issue through streamwise velocity measurements in these three flows with carefully matched Reynolds number and measurement resolution. Results show that mean statistics in the near-wall region collapse well. However, the premultiplied energy spectra of streamwise velocity fluctuations show marked structural differences that cannot be explained by scaling arguments. It is concluded that, while similarities exist at these Reynolds numbers, one should exercise caution when drawing comparisons between the three shear flows, even near the wall.

\section{Introduction}

A review of literature concerning canonical wall-bounded shear flows (pipes, channels and turbulent boundary layers) reveals inconsistencies in regard to the effect of geometry on the structure of turbulence. Most articles implicitly convey the view that pipes, channels and boundary layers are similar in the near-wall region, often with vague caveats. Others are explicit; e.g. Rotta (1962) claims that 'the flow near the wall in pipe and channel flow are the same as in boundary layers'. Alternatively, some suggest that even the mean velocity profiles should be different in pipes/channels as compared to boundary layers (e.g. Wosnik, Castillo \& George 2000). Nevertheless, most agree on the similarity of pipe and channel flows, "because the curvature of the [pipe] wall is nearly zero if seen from points close enough to the surface...' (Tennekes \& Lumley 1972). Hereafter, pipes/channels are referred to as 'internal' geometries, while boundary layers are termed 'external'.

The classical logarithmic formulation for the mean velocity profile (3.1) in a pipe flow is perhaps the most commonly accepted of the concepts extended to boundary layers, notwithstanding the objections by Wosnik et al. (2000). Within the scatter of the data, this extension appears to be valid, although there are ongoing disagreements about the 'universal' constants in the formulation (see Zagarola \& Smits 1998; Nagib \& Chauhan 2008). Further, del Álamo et al. (2004), Jiménez \& Hoyas (2008), Mochizuki \& Nieuwstadt (1996) and Metzger \& Klewicki (2001) show some similarities in higher order streamwise statistics for internal and external flows

$\dagger$ Email address for correspondence: montyjp@unimelb.edu.au 
(note that Jiménez \& Hoyas 2008 observed differences in the wall-normal intensities of the flows). It is also interesting to note that accepting similarity between the flows was important in the first efforts towards understanding the structure of the near-wall flow (Theodorsen 1952; Townsend 1961; Perry \& Chong 1982), due to the lack of reliable turbulent boundary layer data. More recently, new measurement techniques and computational advancement have permitted detailed interrogation of the structure of wall turbulence. For example, using particle image velocimetry, Wu \& Christensen (2006) searched for hairpin heads (spanwise vortical motions) in channels and boundary layers. Based on eddy population figures, they concluded that the flows were structurally similar when $z<0.45 \delta(z$ is distance from the wall and $\delta$ is the outer length scale). Contrarily, Monty et al. (2007) and Hutchins \& Marusic (2007b) found significant structural differences between pipes/channels and boundary layers even in the logarithmic region, based on spanwise structure.

Characterization of the longest energetic modes in wall-turbulence has featured prominently in recent investigations. From single-point hot-wire measurements in pipe flow, Kim \& Adrian (1999) identified a peak or shoulder in the premultiplied energy spectra $\left(k_{x} \phi_{u u}\right)$ at streamwise length scales of more than 14 pipe radii, which they termed Very Large-Scale Motions (VLSM). Here $k_{x}$ is the streamwise wavenumber, and $\phi_{u u}$ is the spectral density of streamwise velocity fluctuations. From a spanwise array of hot-wire measurements, Hutchins \& Marusic (2007a) demonstrated elongated regions of very long positive and negative momentum deficits in the log region of turbulent boundary layers, which they termed 'superstructures'. However, an outer peak in the energy spectra was observed at $\lambda_{x} \approx 6 \delta\left(\lambda_{x}\right.$ is the streamwise wavelength $=$ $\left.2 \pi / k_{x}\right)$, which they attributed to these superstructures. Through similar hot-wire array measurements, Monty et al. (2007) found qualitatively similar events in internal flows, despite the differences in energy spectra evident when comparing results of Kim \& Adrian (1999) and Hutchins \& Marusic (2007a). In a recent, pertinent investigation, Balakumar \& Adrian (2007) investigate the difference in power spectral density between channels and boundary layers. Though differences were noted, they ultimately concluded that the 'large-scale eddies are similar' in internal and external flows.

Hence, from the literature alone, the researcher is left to conclude that internal and external flows have strong similarities near the wall, yet there are clearly important differences. Unfortunately, the nature of the important differences is unclear. It should also be noted that understanding these differences is of great significance at this time as channel flow direct numerical simulations with $R e_{\tau} \gtrsim 1000$ are now readily available; the results of which have been referred to in arguments pertaining to general wall-bounded turbulent flows (e.g. del Álamo et al. 2006; Hutchins \& Marusic 2007a). Furthermore, the aforementioned recent interest in very long flow features (which have been shown in the literature to be geometry dependent) also makes this study especially timely. This investigation aims to clearly illustrate the differences in the three wall-bounded flows through a comparison of carefully matched streamwise velocity measurements.

\section{Facilities}

All facilities are located at Melbourne and have been used in previous investigations. The fully developed pipe and channel flow facilities are detailed in Monty et al. (2007) and the boundary layer wind-tunnel, with $27 \mathrm{~m}$ long working-section, is described in Nickels et al. (2005). The channel and boundary layer are blow-down non-recirculating 


\begin{tabular}{|c|c|c|c|c|c|c|c|c|c|c|c|c|}
\hline \multirow[b]{2}{*}{ Facility } & \multicolumn{5}{|c|}{ Flow conditions } & \multicolumn{3}{|c|}{ Hot-wire details } & \multicolumn{3}{|c|}{ Acquisition details } & \multirow[b]{2}{*}{ Symbol } \\
\hline & $R e_{\tau}$ & $\begin{array}{c}x \\
(\mathrm{~m})\end{array}$ & $\begin{array}{c}U_{\infty} \\
\left(\mathrm{m} \mathrm{s}^{-1}\right)\end{array}$ & $\begin{array}{c}\delta \\
(\mathrm{m})\end{array}$ & $\begin{array}{l}\nu / U_{\tau} \\
(\mu \mathrm{m})\end{array}$ & $\begin{array}{c}l \\
(\mathrm{~mm})\end{array}$ & $\begin{array}{c}d \\
(\mu \mathrm{m})\end{array}$ & $l^{+}$ & $\Delta t_{s}^{+}$ & $\begin{array}{c}f_{s} \\
(\mathrm{kHz})\end{array}$ & $\begin{array}{c}T U_{\infty} / \delta \\
\times 10^{4}\end{array}$ & \\
\hline TBL & 3020 & 5.0 & 12.5 & 0.1003 & 33.2 & 1 & 5.0 & 30 & 0.57 & 24 & 2.25 & 光一 \\
\hline Channel & 3015 & 17.6 & 23.1 & 0.0500 & 16.7 & 0.5 & 2.5 & 30 & 0.55 & 100 & 2.77 & $\square--$ \\
\hline Pipe & 3005 & 17.3 & 24.3 & 0.0494 & 16.4 & 0.5 & 2.5 & 30 & 0.56 & 100 & 2.95 & $\circ-\cdot$ \\
\hline
\end{tabular}

TABLE 1. Parameters for the pipe, channel and turbulent boundary layer (TBL) experiments.

wind-tunnels, while the pipe is a suction facility. Inlet flow to all facilities is conditioned to provide uniform negligibly turbulent conditions at the inlet. This inlet flow is tripped with sandpaper strips. The aspect ratio of the channel is 11.7:1 ensuring minimal sidewall influence. The boundary layer tunnel has a cross-section of $1 \mathrm{~m} \times 2 \mathrm{~m}$, giving a boundary layer height to tunnel width ratio of approximately $16: 1$ at the measurement station ( $5 \mathrm{~m}$ downstream of the trip). Further experimental details are given in table 1.

Experiments in all three facilities were performed at matched friction Reynolds number or Kármán number $R e_{\tau}$, defined as the ratio of the outer to the viscous length scales $\left(=\delta U_{\tau} / \nu\right.$ or $\left.\delta^{+}\right)$. The outer length scale $\delta$ is either the channel halfheight, pipe radius or boundary layer thickness as determined from a modified Coles fit (see Perry, Marusic \& Jones 2002). $U_{\tau}$ is the friction velocity $\left(=\sqrt{\tau_{w} / \rho}\right.$, where $\tau_{w}$ is the wall shear stress and $\rho$ is the density of the fluid) and $v$ is the kinematic viscosity. Throughout the paper, capitalized velocities or overbars indicate time-averaged values. Lowercase velocities indicate the fluctuating component. The superscript + is used to denote viscous scaling of length (e.g. $\left.z^{+}=z U_{\tau} / v\right)$, velocity $\left(U^{+}=U / U_{\tau}\right)$ and time $\left(t^{+}=t U_{\tau}^{2} / \nu\right)$. In order to accurately compare velocity fluctuation statistics, it is necessary to maintain a constant non-dimensional wire length, $l^{+}=l U_{\tau} / \nu$, where $l$ is the length of the etched or active portion of the hot-wire sensor. For all experiments $l^{+}=30 \pm 1$. For pipe and channel measurements, the same hot-wire circuit (custom made) and Dantec 55P15 hot-wire probe (Wollaston wire, $2.5 \mu \mathrm{m}$ diameter, $l=0.5 \mathrm{~mm}$ ) were employed. Since the pipe and channel are roughly of the same dimensions, while the boundary layer is much thicker, at matched $R e_{\tau}$ a longer wire $(5 \mu \mathrm{m}$ diameter Wollaston wire, $l=1 \mathrm{~mm}$ ) was required for the boundary layer measurements to maintain a constant non-dimensional wire length. An $A A$ Labs $A N-1003$ hot-wire circuit and a Dantec 55P05 probe were used in the boundary layer. In all cases, hot-wires were heated with an overheat ratio of 1.8 and the systems had a frequency response of at least $50 \mathrm{kHz}$, determined by injecting a square wave into the circuit. Hot-wire voltage was sampled for a minimum of $20000 \delta / U_{\infty} \mathrm{s}$, where $U_{\infty}$ is the mean centreline or free stream velocity. Sampling rate was always greater than $U_{\tau}^{2} / v \mathrm{~Hz}$ (sample interval $\Delta t^{+}<1$ ) to ensure all significant energy containing motions were temporally resolved.

\section{Mean statistics}

The mean velocity profile scaled with inner variables $\left(U_{\tau}\right.$ and $\left.\nu\right)$ for all three flows is presented in figure 1(a). Excellent collapse of the data is observed up to the edge of the logarithmic region $(z<0.15 \delta)$ and arguably up to $z \approx 0.25 \delta$. Note that $U_{\tau}$ is calculated from pressure drop for the internal geometries, allowing unambiguous comparison of 

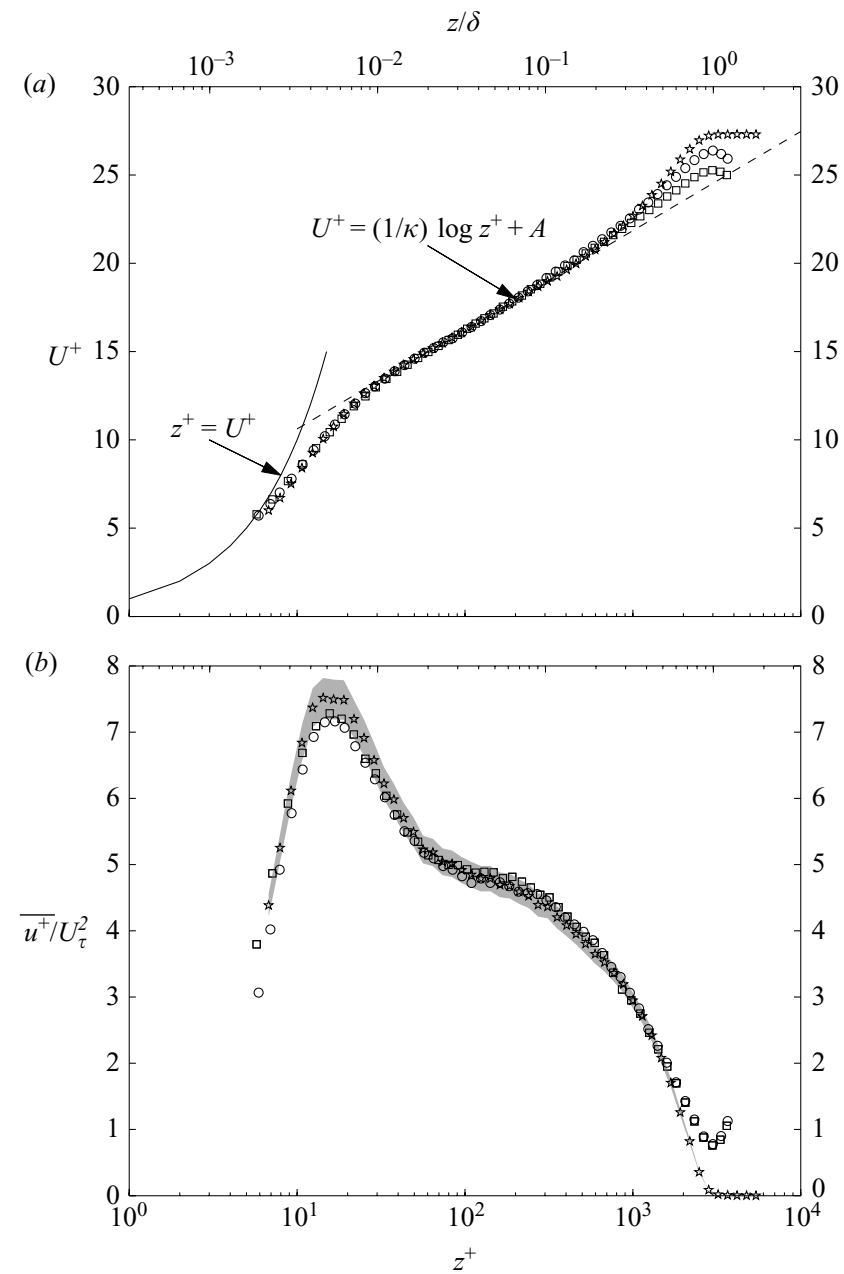

FIGURE 1. (a) Mean velocity profiles and (b) associated broadband turbulence intensity for ( $\square$ ) channel, (O) pipe and ( $)$ turbulent boundary layer at matched $R e_{\tau}$. Solid line in $(a)$ shows $U^{+}=z^{+}$. Dashed line shows $U^{+}=(1 / \kappa) \ln \left(z^{+}\right)+A$ (where $\kappa=0.41$ and $\left.A=5.0\right)$. Shading in (b) shows $\pm 4 \%$ variation from boundary layer data.

the profiles of pipe and channel flows. For the boundary layer, the Clauser method was employed due to the absence of a more reliable alternative. Log law (3.1) constants were chosen to be $\kappa=0.41$ and $A=5.0$ (oil-film interferometry measurements have been performed in this tunnel, giving similar $U_{\tau}$ values - within $\pm 1 \%$ of Clauser; it is noted, however, that this technique has its own inaccuracies that are beyond the scope of this study). Well beyond the log region, the profiles are clearly different, as previously discussed by Monty et al. (2007). In one of the first detailed comparisons of internal and external flows, Schubauer (1954) also highlights the differences in mean velocity profiles in the outer-flow region (although he rightly points out that the general trends are strikingly similar for such different flow geometries).

The traditional logarithmic law, valid in the range $100<z^{+}<0.15 R e_{\tau}$,

$$
U^{+}=\frac{1}{\kappa} \ln z^{+}+A,
$$


$z / \delta$

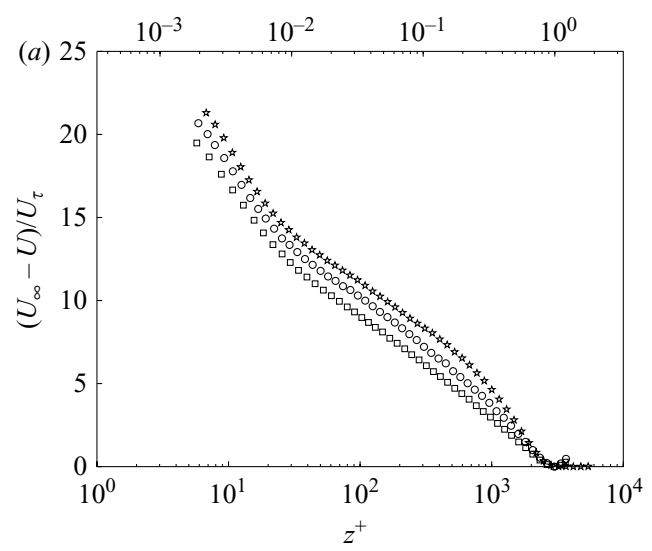

$z / \delta$

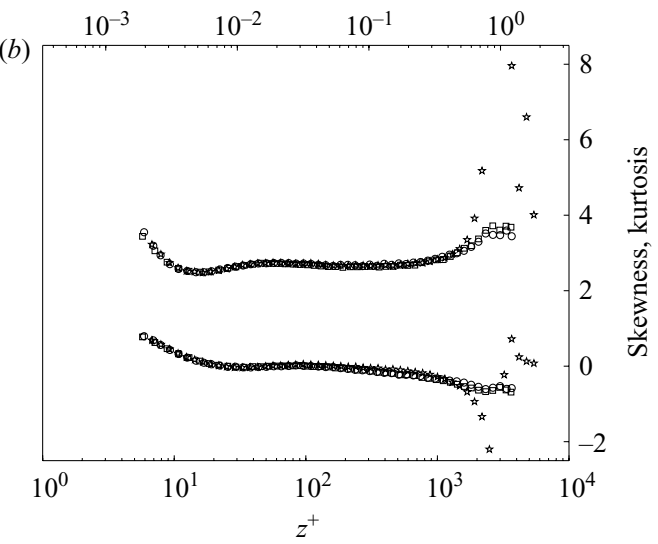

Figure 2. (a) Velocity deficit plots for the three test geometries. (b) Skewness (lower profiles) and kurtosis (upper) profiles. Symbols as in figure 1.

is also shown in figure 1(a), where $\kappa$ and $A$ are supposedly universal constants. Given the limited Reynolds number of the present experiments (meaning limited logarithmic region), it is not possible to draw conclusions about the values of these universal constants, other than to say $\kappa$ and $A$ appear nominally the same for all three flows, within experimental error. The mean velocity deficit should also have a logarithmic nature,

$$
\frac{U_{\infty}-U}{U_{\tau}}=-\frac{1}{\kappa} \ln \left(\frac{z}{\delta}\right)+B
$$

where $B$ is non-universal, depending only on flow geometry. In fact, $B$ is simply proportional to the wake strength. The data in figure $2(a)$ confirm this as the profiles are clearly shifted upwards in order of wake strength, having similar slopes in the log region.

Moving to higher order statistics, the viscous scaled variance of streamwise velocity fluctuations is shown in figure $1(b)$. Within experimental error the three flows are in agreement up to $z \approx 0.5 \delta$. The grey shaded region in figure $1(b)$ represents the boundary layer variance $\pm 4 \%$, which may be considered an approximate error bound, based on the hot-wire measurement error $(\approx \pm 1 \%$ in $U)$ and the error in determining $U_{\tau}$ from the mean velocity profile (at least $\pm 1 \%$ ). The largest differences, occurring at $z^{+} \approx 15$, are within this approximate bound and perhaps should not be concluded significant at this stage. Certainly, the peak turbulence intensities are all within the scatter seen in the literature (e.g. Metzger \& Klewicki 2001; Hutchins et al. 2009). Comparing pipe and channel data, close similarity is observed right across the flows, even in the core region, where one would expect to see effects owing to the radically different geometries. Also interesting is the extent of agreement between the boundary layer and internal flows, up to $z \approx 0.5 \delta$, well beyond the collapse of mean velocity (see figure 1a). Klebanoff (1954) and Schubauer (1954) point out that the intermittent region of the boundary layer $(z>0.4 \delta)$ contains distinct regions of turbulent flow and potential flow. Dividing the turbulent kinetic energy by the intermittency factor, Schubauer illustrates that the boundary layer kinetic energy follows the same distribution as the internal geometry right to the edge of the layer (Klebanoff 1954 also discusses the similarity between rescaled kinetic energy of pipes and boundary layers). From this he inferred that the outer-flow structure must be 
the same for internal and external geometries, with the only difference being the intermittent periods of irrotational flow in the boundary layer.

Figure 2(b) displays the skewness and kurtosis plotted against scaled distance from the wall. The trends are similar to the turbulence intensity data: both internal and external flows have very similar skewness and kurtosis up to $z \approx 0.5 \delta$, while these statistics for the pipe and channel flows are almost identical throughout the flow (it could be argued that the boundary layer skewness exhibits a slightly different trajectory for $z^{+} \gtrsim 200$ ). Beyond $z \approx 0.5 \delta$, the boundary layer data deviate rapidly due to increasing intermittency.

\section{Energy spectra}

From a comparison of the prominent recent work on the largest-scale motions of turbulent pipe, channel and boundary layer flows (Kim \& Adrian 1999; del Álamo et al. 2006; Guala, Hommema \& Adrian 2006; Hutchins \& Marusic 2007a), one point is clear: the large-scale peak in energy spectra of internal flows occurs at significantly longer wavelengths than that in boundary layers. Precisely in which part of the flow this difference occurs is not clear; nor is it obvious whether pipes and channels share similar energy spectra. In an attempt to provide an overall picture of the energy distribution, contour maps of energy contribution are plotted against wall distance and wavelength in figure $3(a-f)$. In all the figures, the contour and colour levels are the same (colour indicates the magnitude of the premultiplied spectra $k_{x} \phi_{u u}^{+}$as given by the scale on the right of the figure). This style of spectra presentation was used by Hutchins \& Marusic $(2007 a, b)$, the latter providing a detailed description of how these plots are constructed. Figures $3(a), 3(c)$ and $3(e)$ are shown with logarithmic axes. Clearly, the qualitative, overall view of the three flows is similar, in agreement with Balakumar \& Adrian (2007). There is a highly energetic peak near the wall (centred at $z^{+} \approx 15, \lambda_{x}^{+} \approx 1000$ and marked with a white cross). This 'inner peak' is due to the near-wall cycle of streaks and quasi-streamwise vortices. The peak energy shifts to larger wavelengths as we move away from the wall. As noted by Hutchins \& Marusic $(2007 b)$, there is a secondary peak in the boundary layer spectra map at $z \approx 0.06 \delta$, corresponding to superstructures of wavelength, $\lambda_{x} \approx 6 \delta$ (Hutchins \& Marusic 2007a observed from flow visualization that superstructures can be considerably longer than $6 \delta$, but meander in the spanwise direction so that their signature in the energy spectrum is found at shorter length scales). This peak is marked with a black cross in all spectra maps (figure $3 a-f$ ). While the energy peak of the boundary layer moves to smaller wavelengths for $z>0.06 \delta$, this is not the case for pipes and channels. Firstly, in comparison with the boundary layer, higher energy is observed in the longer wavelength modes of pipe/channel flows from the beginning of the log region (notice the contours cut through the top of the figure for internal flows, but not for the boundary layer). On closer inspection, there appears to be an increasing difference in length between the longest energetic structures in the internal and external flows as we move away from the wall. Well into the outer-flow region $(z \gtrsim 0.3 \delta)$, the pipe/channel plots show a 'double-hump' in the contours, indicating two dominant modes, whereas the boundary layer has only one as noted by Balakumar \& Adrian (2007). The single dominant mode in the boundary layer spectra for $z>0.3 \delta$ coincides with the shorter energetic mode in the internal flows. This mode was identified by Adrian, Meinhart \& Tomkins (2000) as the large-scale motion (LSM), having length $\sim 2-3 \delta$. Guala et al. (2006) also commented on the observed similarity of these large-scale motions in the outer-flow regions of pipes, channels and boundary layers. The second peak in the 


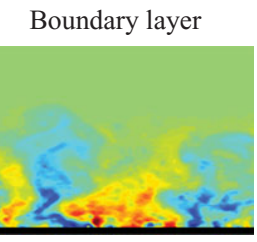

Channel
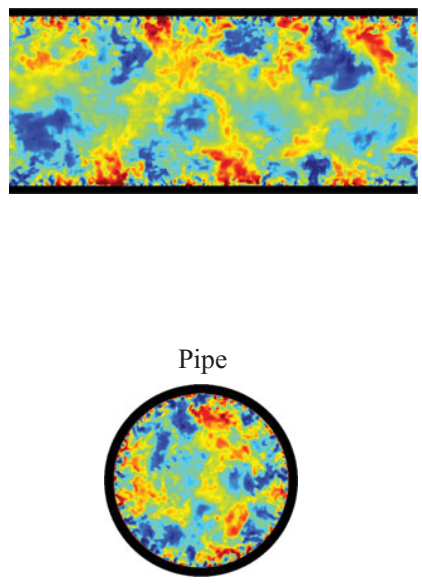

(a)

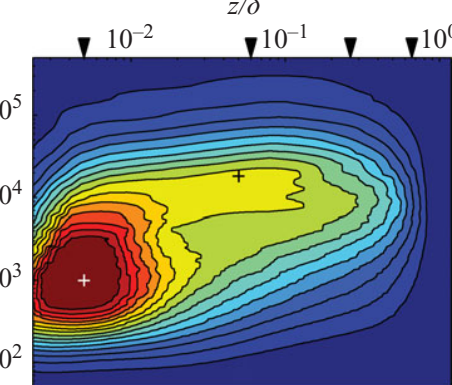

(c)

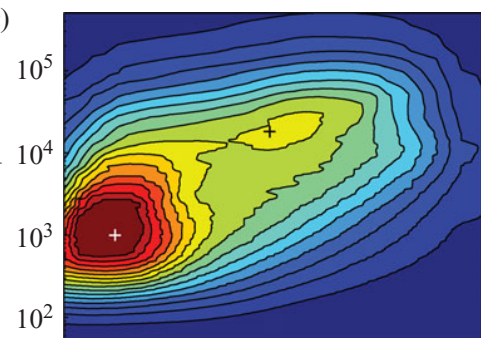

(e)

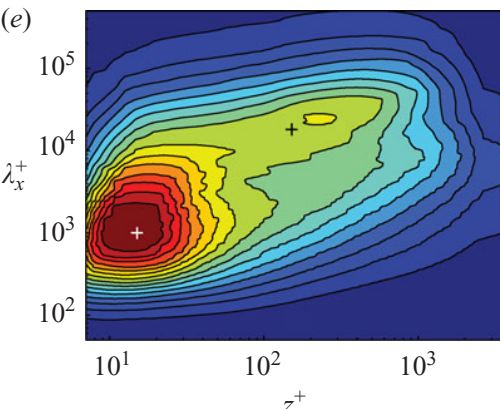

$z / \delta$

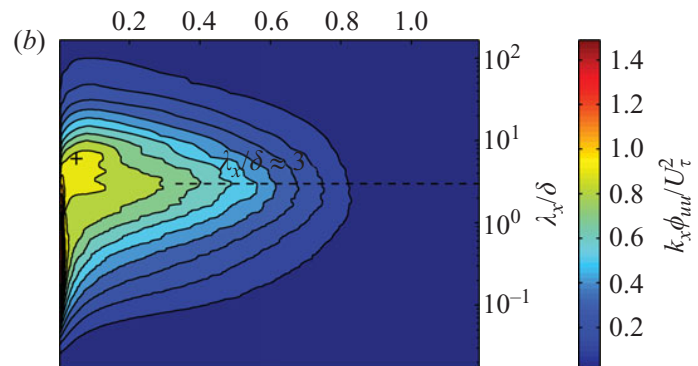

$(d)$

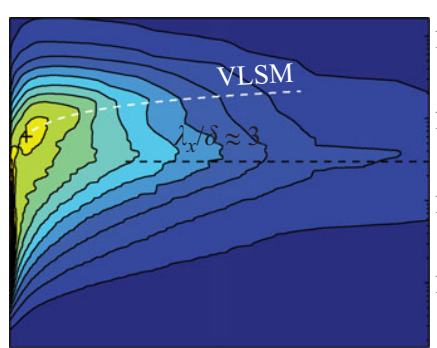

$10^{2}$

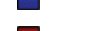

1.4

1.2

1.0

100 ₹

$10^{-1} \quad 0.4$

0.2

(f)

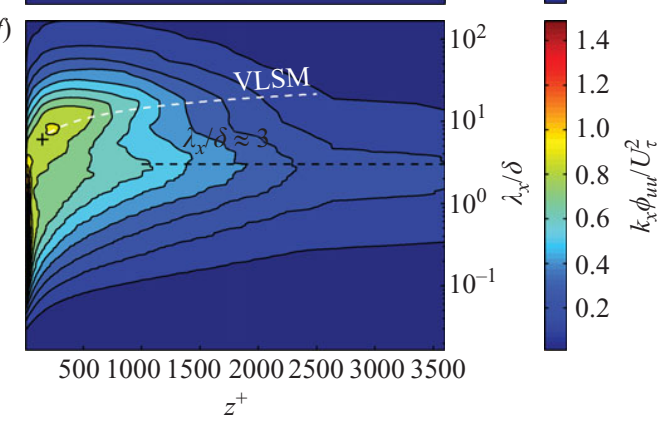

FIGURE 3. Maps of premultiplied energy spectra of streamwise velocity fluctuation as a function of energetic length scale $\left(\lambda_{x}\right)$ and distance from the wall $(z)$. Figures show (from top to bottom) turbulent boundary layer $(a, b)$, channel $(c, d)$ and pipe $(e, f)$, respectively. Right-hand figures $(b, d, f)$ have a linear $x$-axis. Left-hand figures show representative cross-sections of flow geometry for illustrative purposes, from (top) Hutchins, Hambleton \& Marusic (2005) and (middle \& bottom) Tsubokura (2005). 

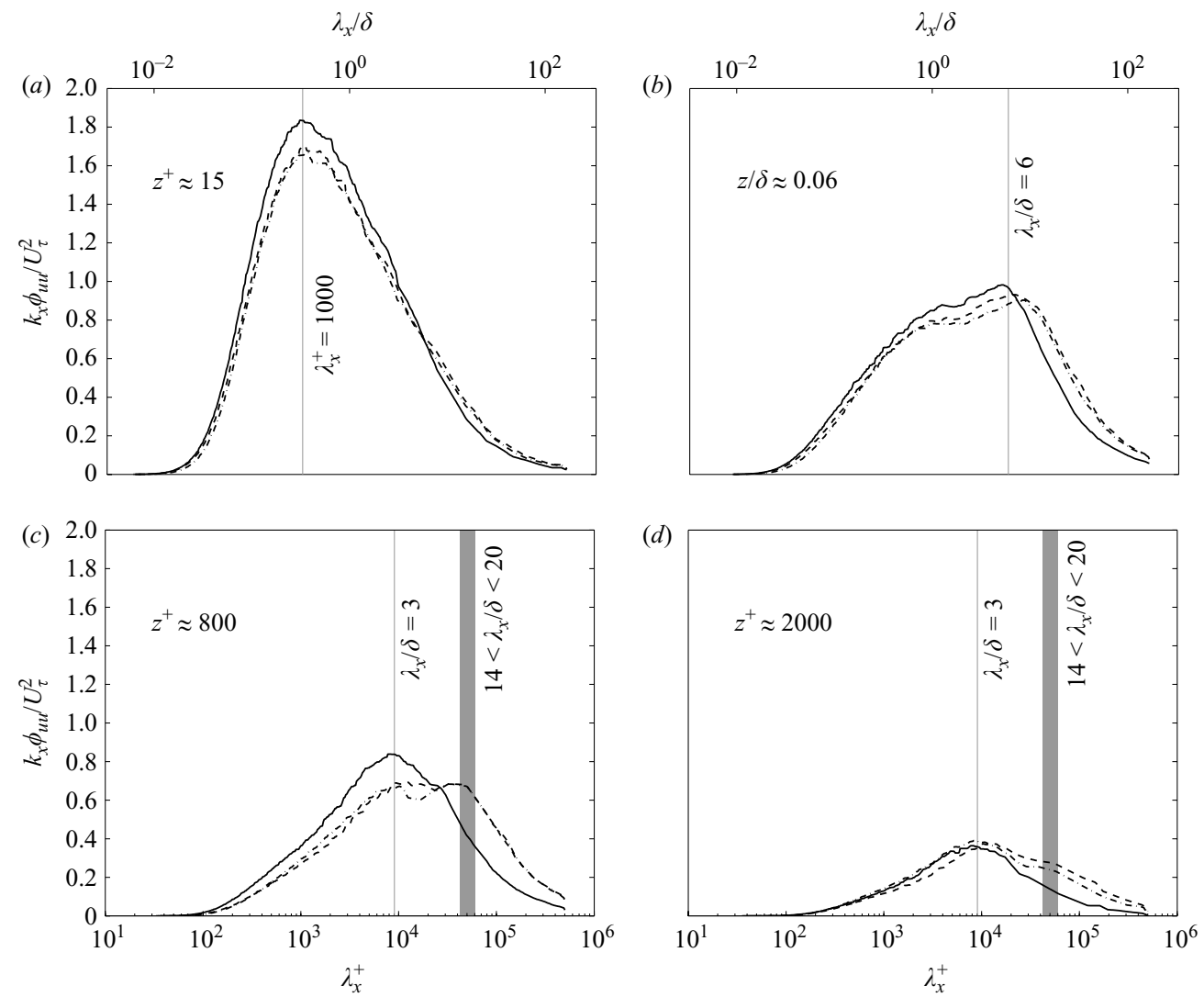

FIGURE 4. Comparisons of premultiplied energy spectra of $u$ fluctuations at $(a) z^{+} \approx 15$ $(z / \delta \approx 0.005) ;(b) z^{+} \approx 180(z / \delta \approx 0.060) ;(c) z^{+} \approx 800(z / \delta \approx 0.265) ;(d) z^{+} \approx 2000(z / \delta \approx 2 / 3)$ for $(-)$ boundary layer, $(--)$ channel and $(-\cdot-)$ pipe.

energy spectra of pipes/channels, however, occurs at much longer wavelengths than the first $\left(14<\lambda_{x}<20\right.$, as highlighted in figure $\left.4 d\right)$ and much longer than any energetic mode in the boundary layer. Note that it is difficult to quantify the wavelength of these motions beyond $z \approx 0.33 \delta$, since they grow with wall distance while decaying in energy. Eventually, the 'peak' in energy formed by these motions closer to the wall $(z \approx 0.1-0.33 \delta)$ becomes little more than the shoulder of a plateau (e.g. compare figures $4 c$ and $4 d$ ).

It is now evident that the most distinguishing features of the three spectra contour maps are found in the outer region. Plotting these figures with logarithmic absiccsae, however, focuses attention on the near-wall region. Therefore, figures $3(b), 3(d)$ and $3(f)$ are provided, displaying the same information as figures $3(a), 3(c)$ and $3(e)$, only with linear abscissae, focusing attention on the outer region of the flow. For visual guidance, lines showing the dominant large-scale mode in the outer region of the boundary layer, $\lambda_{x}=3 \delta$, and the second dominant mode in the pipe and channel are shown (broken white line). This second, longer mode is that identified by Kim \& Adrian (1999) as the VLSM. The VLSM growth appears to roughly follow a power law,

$$
\frac{\lambda_{x}}{\delta}=23\left(\frac{z}{\delta}\right)^{3 / 7},
$$


which approximately agrees with the peak wavelength data given in figure 5 of Kim \& Adrian (1999). Regardless of the growth rate, there are obvious and important differences between boundary layer superstructures and internal flow VLSMs. The superstructures do not persist beyond the log region of the boundary layer, rather a rapid shortening of the most energetic structures occurs, leading quickly to the domination of $\lambda_{x} \approx 3 \delta$ structures throughout most of the outer region. Conversely, the wavelength of VLSMs continues to increase, arguably as far as $z \approx 0.7 \delta$, while their energy magnitudes decrease as we move further into the core region.

There are no obvious or significant differences between the energy spectra for pipes and channels in the spectral representations of figure 3, even in the core. This is consistent with the agreement discussed previously for the mean statistical analysis of $\S 3$. Although it should be noted that Balakumar \& Adrian (2007) show differences between the Reynolds shear stress associated with VLSMs of pipes and channels. While the mean statistics of the internal and external flows are also similar up to $z \approx 0.5 \delta$, the energy spectra are clearly very different, even near the wall. This is more easily observed from figure 4, where premultiplied spectra are compared at a selection of $z$-coordinates; coordinates are marked with arrows on top of figure $3(a)$. Firstly, figure $4(a)$ shows the energy spectra at the near-wall peak in turbulence intensity the most energetic region of the flow. The insignificance of pipe curvature very close to the wall (as suggested by Tennekes \& Lumley 1972) is strongly supported by the similarities in energy spectra exhibited by both internal flows at this wall distance. However, the external flow case is not so similar; although the overall intensity is slightly higher for the boundary layer, it is clear that the shape of the spectra is different for internal and external flows. The discrepancy is in the largest scales, where the internal flows exhibit more energy than the boundary layer for $\lambda_{x} \gtrsim 7 \delta$. This difference is expected, since this very large-scale energy near the wall is effectively the footprint (Hutchins \& Marusic 2007a) of the largest modes that inhabit the outer-flow regions, which figure 3 has clearly shown are longer for internal flows.

Figures $4(b)$ and $4(c)$ display energy spectra at two locations in the outer-flow region and it is reminded that the area under the curves is proportional to the scaled turbulence intensity. Figure $4(c)$ clearly shows that the structure of the internal and external flows is fundamentally different, most notably in the largest scales. Throughout this paper, the outer length scale has been presumed as is generally accepted in the literature. Modification to $\delta$ without physical basis would permit collapse of the spectra for a certain range of wavelengths (although there will be an adverse effect on outer-scaling collapse of statistics profiles shown in $\S 3$ ). However, it is not possible to collapse the entire large wavelength regime (i.e. $\lambda_{x}>3 \delta$, where $\delta$ is the traditional boundary layer thickness) of the energy spectra in all three flows. This is because there is no location in the boundary layer flow where the structure is bimodal with dominant modes of $3 \delta$ and $\sim 15 \delta$. No outer-scaling argument, therefore, can explain the noted differences in these flows. According to figure $1(b)$, the turbulence intensity is essentially equal for all flows at the wall distances pertaining to figures $4(b)$ and $4(c)$. This implies that the different shapes of the spectra result from a redistribution of energy from the smaller scales dominant in boundary layers to longer wavelengths in the pipe/channel. Although it is difficult to comment on mechanisms for this behaviour, it is clear that the conditions in pipes/channels must permit the very large modes to persist further from the wall than in boundary layers (in which they are largely constrained to the log region).

Finally, since we are most interested in the difference between flows, a plot of the difference in energy between pipes/channels and boundary layers is warranted. 


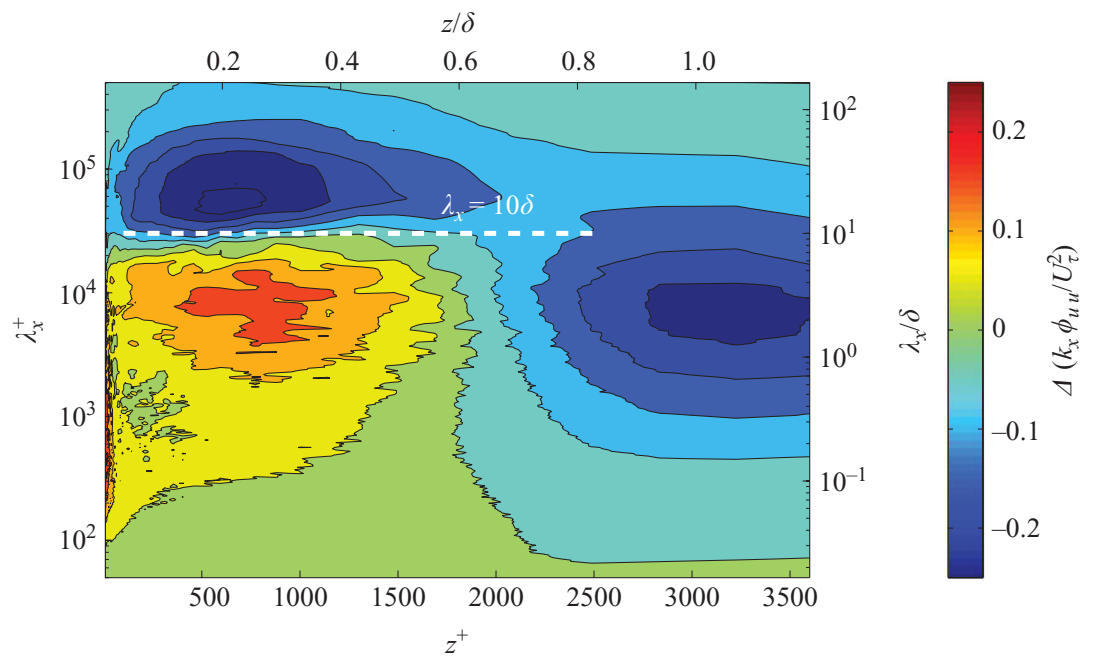

FIGURE 5. Contours of $\left|k_{x} \phi_{u u}^{+}\left(z, \lambda_{x}\right)\right|_{b l}-\left|k_{x} \phi_{u u}^{+}\left(z, \lambda_{x}\right)\right|_{p i p e}$. The difference between the energy spectra of the pipe and boundary layer as a function of $z$ and $\lambda_{x}$.

For this purpose, we may assume that pipes and channels have the same energy distribution throughout the flow. Hence, figure $3(f)$, the energy map for pipe flow, was subtracted from $3(b)$, the map for boundary layers, with the result displayed in figure 5. Contours now represent the scaled energy difference between pipes/channels and boundary layers; regions of blue shading correspond to higher energy in the pipe/channel and red to higher energy in the boundary layer. A linear abscissa is again used to highlight differences in the log region and beyond. Two obvious demarcations are observed. For very long wavelengths, $\lambda_{x}>10 \delta$, there is significantly more energy for internal flows than that of the boundary layers; the opposite is true for $\lambda_{x}<10 \delta$. Also, far from the wall $(z \gtrsim 0.6)$, the geometrical freedom of the boundary layer is highlighted as energy ultimately decays to zero at the edge of the boundary layer, while the internal flows remain turbulent through the core.

\section{Conclusions}

Through a simple comparison of mean statistics and energy spectra for pipe, channel and boundary layer flows at matched Kármán number $\left(\delta^{+}\right)$, the similarities and differences between these flows have been clearly documented. Within experimental error, the inner-scaled mean velocity is identical for all three flows in the region $z<0.25 \delta$ as expected. The agreement extends much further for the higher order statistics, which display collapse up to $z=0.5 \delta$ (at least).

After performing Fourier analyses of the data, surprising agreement was observed between the structure of pipe and channel flow throughout the flow. However, there are obvious important modal differences between channels/pipes and boundary layers, not only in the outer/core region, but right down to the wall. The difference is in the largest energetic scales, which are much longer in pipes/channels. Although the large-scale phenomena have been shown to be qualitatively similar (Hutchins \& Marusic 2007a; Monty et al. 2007), their contributions to the energy continues to move to longer wavelengths with distance from the wall in internal flows. The opposite occurs in boundary layers, where outer-flow structures shorten very rapidly beyond 
the $\log$ region. To re-word this conclusion using terminology of recent literature: VLSMs in internal flows should not be confused with superstructures in boundary layers; qualitatively the structures are similar, however, the VLSM energy in internal flows resides in larger wavelengths and at greater distances from the wall than superstructures in boundary layers. Furthermore, for $z<0.5 \delta$ the different energy distributions in internal and external flows occur in regions where the turbulence intensity (streamwise kinetic energy) is equal. This result suggests that all three flows might be of a similar type structure, with energy simply redistributed from shorter to longer scales for the pipe and channel flow cases. Whether the quantitative differences are due to the interaction of the opposite wall in internal flows, or the intermittency of the outer region in boundary layers remains uncertain.

Finally, it is expected that the observed large-scale differences between pipes/channels and boundary layers will be more obvious at higher Reynolds numbers as Hutchins \& Marusic (2007a) have shown that the magnitude of the energy contribution of superstructures increases with Reynolds number.

The authors are grateful for the financial support of the Australian Research Council through projects DP0556629, FFD0668703 and DP0663499.

\section{REFERENCES}

Adrian, R. J., Meinhart, C. D. \& Tomkins, C. D. 2000 Vortex organization in the outer region of the turbulent boundary layer. J. Fluid Mech. 422, 1-54.

del Álamo, J. C., Jiménez, J., Zandonade, P. \& Moser, R. D. 2004 Scaling of the energy spectra of turbulent channels. J. Fluid Mech. 500, 135-144.

del Álamo, J. C., Jiménez, J., Zandonade, P. \& Moser, R. D. 2006 Self-similar vortex clusters in the turbulent logarithmic region. J. Fluid Mech. 561, 329-358.

BAlakumar, B. J. \& AdRian, R. J. 2007 Large- and very-large-scale motions in channel and boundary layer flows. Phil. Trans. R. Soc. A 365 (1852), 665-681.

Guala, M., Hommema, S. E. \& Adrian, R. J. 2006 Large-scale and very-large-scale motions in turbulent pipe flow. J. Fluid Mech. 554, 521-542.

Hutchins, N., Hambleton, W. T. \& Marusic, I. 2005 Inclined cross-stream stereo particle image velocimetry measurements in turbulent boundary layers. J. Fluid Mech. 541, 21-54.

Hutchins, N. \& Marusic, I. $2007 a$ Evidence of very long meandering features in the logarithmic region of turbulent boundary layers. J. Fluid Mech. 579, 1-28.

Hutchins, N. \& Marusic, I. 2007b Large-scale influences in near-wall turbulence. Phil. Trans. R. Soc. A 365 (1852), 647-664.

Hutchins, N., Nickels, T. B., Marusic, I. \& Chong, M. S. 2009 Hot-wire spatial resolution issues in wall-bounded turbulence. J. Fluid Mech., in press.

JimÉNEZ, J. \& HoYAS, S. 2008 Turbulent fluctuations above the buffer layer of wall-bounded flows. J. Fluid Mech. 611 (1), 215-236.

Kim, K. C. \& Adrian, R. J. 1999 Very large-scale motion in the outer layer. Phys. Fluids 11 (2), 417-422.

KLEBANOFF, P. S. 1954 Characteristics of turbulence in a boundary layer with zero pressure gradient. Tech. Rep. TN3178. NACA.

Metzger, M. M. \& KLewicki, J. C. 2001 A comparative study of near-wall turbulence in high and low Reynolds number boundary layers. Phys. Fluids 13, 692-701.

MochizUKi, S. \& NieuwstadT, F. T. M. 1996 Reynolds-number-dependence of the maximum in the streamwise velocity fluctuations in wall turbulentce. Exp. Fluids 21, 218-226.

Monty, J. P., Stewart, J. A., Williams, R. C. \& Chong, M. S. 2007 Large-scale features in turbulent pipe and channel flows. J. Fluid Mech. 589, 147-156.

Nagib, H. \& Chauhan, K. A. 2008 Variations of von Kármán coefficient in canonical flows. Phys. Fluids 20 (101518). 
Nickels, T. B., Marusic, I., Hafez, S. \& Chong, M. S. 2005 Evidence of the $k_{1}^{-1}$ law in a high-reynolds-number turbulent boundary layer. Phys. Rev. Lett. 95 (074501).

Perry, A. E. \& Chong, M. S. 1982 On the mechanism of wall turbulence. J. Fluid Mech. 119, $173-217$.

Perry, A. E., Marusic, I. \& Jones, M. B. 2002 On the streamwise evolution of turbulent bundary layers in arbitrary pressure gradients. J. Fluid Mech. 468, 61-91.

RotTA, J. C. 1962 Turbulent boundary layers in incompressible flow. Prog. Aero. Sci. 2, 1-219.

Schubauer, G. B. 1954 Turbulent processes as observed in boundary layer and pipe. J. Appl. Phys. 25, 188-196.

Tennekes, H. \& Lumley, J. L. 1972 A First Course in Turbulence. Massachusetts Institute of Technology.

Theodorsen, T. 1952 Mechanism of turbulence. In Proceedings of Second Midwestern Conference on Fluid Mechanics. Ohio State University.

Townsend, A. A. 1961 Equilibrium layers and wall turbulence. J. Fluid Mech. 11, 97-120.

TsuboKURA, M. 2005 LES study on the large-scale motions of wall turbulence and their structural difference between plane channel and pipe flows. In Proceedings of Fourth International Symposium on Turbulence and Shear Flow Phenomena, TSFP4, Willamsburg, Virginia.

Wosnik, M., Castillo, L. \& George, W. K. 2000 A theory for turbulent pipe and channel flows. J. Fluid Mech. 421, 115-145.

Wu, Y. \& Christensen, K. T. 2006 Population trends of spanwise vortices in wall turbulence. J. Fluid Mech. 568, 55-76.

Zagarola, M. V. \& Smits, A. J. 1998 Mean-flow scaling of turbulent pipe flow. J. Fluid Mech. 373, 33-79. 


\section{University Library}

\section{- M M N E R VA A gateway to Melbourne's research publications}

Minerva Access is the Institutional Repository of The University of Melbourne

Author/s:

Monty, JP;Hutchins, N;Ng, $\mathrm{HCH}$;Marusic, I;Chong, MS

Title:

A comparison of turbulent pipe, channel and boundary layer flows

Date:

2009-08-10

Citation:

Monty, J. P., Hutchins, N., Ng, H. C. H., Marusic, I. \& Chong, M. S. (2009). A comparison of turbulent pipe, channel and boundary layer flows. JOURNAL OF FLUID MECHANICS, 632, pp.431-442. https://doi.org/10.1017/S0022112009007423.

Publication Status:

Published

Persistent Link:

http://hdl.handle.net/11343/32989 